\title{
PROPIEDADES VIBRACIONALES DE CARBUROS DE MOLIBDENO NANOESTRUCTURADOS
}

\author{
Vibrational properties of nanostructured molybdenum carbides
}

\section{EPISTEMUS}

ISSN: 2007-8196 (electrónico)

ISSN: 2007-4530 (impresa)

\section{Luis Iván Serrano Corrales 1 \\ Keren Hapuc Gutiérrez Acosta 2 \\ Ana Bertha López Oyama 3 \\ Rogelio Gámez Corrales 4 \\ Felipe Barffuson Domínguez 5}

Recibido: 27 de marzo de 2015,

Aceptado: 14 de marzo del 2016

Autor de Correspondencia:

I.Q. Luis Ivan Serrano Corrales

Correo: luisiv.sc@gmail.com

\section{Resumen}

En este trabajo se hace un estudio de las propiedades vibracionales de espectroscopía micro Raman de carburos de molibdeno y sus correlaciones con los tamaños de fronteras de grano en función del tiempo de molienda, en atmosfera de gas de argón, y manteniendo la velocidad de molienda constante. La técnica de molienda mecánica de alta energía permitió mantener un control del tamaño de frontera de grano, la cual mostró, en leyes de potencia, dos regiones en función del tiempo de molienda. Mientras que por medio de espectroscopía micro Raman, se observaron cambios en los valores de las bandas principal $\left(819.5 \mathrm{~cm}^{-1}\right)$ y secundarias $(894.0 \mathrm{y}$ $778.7 \mathrm{~cm}^{-1}$ ). Las tres bandas estudiadas presentan un comportamiento línea en función del tiempo de molienda. Estos cambios que se presentan en las vibraciones Raman de estas bandas pudieron correlacionarse directamente con el tamaño de grano observado por microscopía óptica y por consecuencia del área superficial de las micropartículas de carburo de molibdeno. Las vibraciones en las bandas Raman, corresponden a cambios en las bandas características del $\mathrm{Mo}_{2} \mathrm{C}$ así como apariciones de las bandas relacionadas con las vibraciones $\mathrm{O}-\mathrm{Mo}-\mathrm{O}$.

Palabras clave: carburos nanoestructurados, metales de transición, nanomateriales, microscopía óptica, espectroscopía Raman.

\section{Abstract}

This paper presents a study of the properties micro Raman spectroscopy vibrations of nanostructured molybdenum carbides and their correlations with the size of grain boundaries in function of the time of mechanical milling, in argon gas atmosphere is made, and maintaining speed milling constant. The technique of high-energy ball milling control enabled a grain boundary size, which showed, in power laws, two regions in terms of milling time. While micro Raman through spectroscopy, changes were observed in the values of the main bands $\left(819.5 \mathrm{~cm}^{-1}\right)$ and secondary $\left(894.0\right.$ and $\left.778.7 \mathrm{~cm}^{-1}\right)$. The three bands studied show a linear behavior. These changes occurring in the Raman vibration of these bands could be directly correlated with the grain size observed by optical microscopy and consequently the surface area of the micro particles of molybdenum carbide. The vibrations in the Raman bands correspond to changes in the characteristic bands of $\mathrm{Mo}_{2} \mathrm{C}$ and appearances of the bands related to the bond O-Mo-O.

Keywords: Nanostructured carbides, transition metals, nanomaterials, optical microscopy, Raman spectroscopy. 


\section{INTRODUCCIÓN}

Los carburos de metales de transición fueron descubiertos a finales del siglo XIX como producto de mezclarse polvos de tungsteno con carbono a altas temperaturas, el resultado fue un material muy duro y resistente al desgaste (carburo de tungsteno). Actualmente, se sabe que la interacción del carbono con un metal de transición de los grupos IV-VI B de la tabla periódica produce estos carburos metálicos. Para diversos metales de transición, la incorporación del carbono incluso en pequeñas cantidades puede cambiar drásticamente sus propiedades físicas, ópticas, electrónicas y químicas. Sin embargo, una propiedad que resulta de gran interés es la actividad catalítica, al ser utilizados en reacciones químicas, como lo es el caso de los procesos de denitrogenización. Esta propiedad de catalizar, se encuentra estrechamente relacionada al tamaño volumétrico, ya que la relación área superficial/volumen permite tener una mayor área de contacto entre los reactivos a catalizar. La obtención de carburos con mayor área superficial, en las últimas décadas, ha atraído la atención en relación a sus propiedades catalíticas [1]. La actividad catalítica del carburo de molibdeno se ha encontrado fuertemente relacionada con la estructura cristalina y sobre todo de su superficie y composición elemental, la cual en gran medida depende del método de síntesis. Los carburos de metales de transición producidos por los métodos convencionales usualmente no presentan alta actividad catalítica, este problema es superado cuando los carburos son nanoestructuras [2].

Molienda mecánica es una técnica experimental que permite obtener materiales a escala nanométrica, donde la activación mecánica o mecano-síntesis es inducida por el golpeteo de las bolas o balines que se encuentran en el molino planetario. Pudiéndose obtener nuevas fases cristalinas y/o composiciones químicas en carburos metálicos que poseen nuevas propiedades a nivel macroscópico.

En este trabajo se estudia la composición química y estructural de carburos de molibdeno a diferentes tiempo de molienda, por medio de la técnica experimental de espectroscopía micro Raman, comúnmente utilizada en caracterización de materiales, y más recientemente en nanomateriales.

Así como también se llevó a cabo la correlación de los modos normales de vibración y el tamaño de fronteras de grano, utilizando la técnica experimental de microscopía óptica.

\section{DETALLES EXPERIMENTALES}

Los carburos de molibdeno utilizados en este trabajo presentan una pureza del $95 \%$ de la marca Sigma-Aldrich y fueron utilizados sin previa purificación o tratamiento alguno. Se pesaron 6 gramos de carburo de molibdeno los cuales fueron depositados en el tazón de molienda, de un molino planetario Fritsch modelo Pulverissette 7 junto con los elementos moledores (bolas de acero). En todo este trabajo, la molienda del material en polvo se llevó a cabo utilizando una velocidad constante de rotación de $450 \mathrm{rpm}$, bajo atmósfera de gas inerte. Una vez iniciada la molienda el proceso se interrumpió en varias ocasiones, a las 8,12 , $24,36,48,72$ y 96 horas para extraer una pequeña porción de la muestra a los diferentes intervalos y al mismo tiempo para evitar suministrar demasiada energía cinética al sistema a través del golpeteo de los balines con la muestra, y evitando de esta manera se lleve a cabo una transición de fase de los carburos de molibdeno, por lo que se pretende mantener la misma estructura cristalina. Se caracterizaron las muestras extraídas a los distintos tiempos de molienda y se determinó el tamaño de grano de carburo de molibdeno en cada una de las etapas, utilizando la técnica micro Raman y microscopía óptica respectivamente.

\section{RESULTADOS Y DISCUSIÓN}

La figura 1 (parte superior), muestra el espectrograma micro Raman característico de carburos de molibdeno $\mathrm{Mo}_{2} \mathrm{C}$, obtenido a presión atmosférica y a temperatura ambiente $\left(26^{\circ} \mathrm{C}\right)$. Las mediciones se llevaron a cabo utilizando vectores de onda entre 100 y $2000 \mathrm{~cm}^{-1}$, donde se pueden apreciar (Figura 1), tres regímenes bien definidos. A altos valores de vector de onda (1200 a 2000 $\mathrm{cm}^{-1}$ ) se presenta un pico con muy baja intensidad, a $1580 \mathrm{~cm}^{-1}$, el cual corresponde a los modos normales de grafito, y es identificada en la literatura como la banda G, es como consecuencia de las vibraciones tangenciales. A valores intermedios $\left(400-1200 \mathrm{~cm}^{-1}\right)$ se presenta la región correspondiente a los óxidos metálicos, carburos metálicos y oxi-carburos metálicos. Y finalmente a valores de vectores de onda más pequeños $\left(100-400 \mathrm{~cm}^{-1}\right)$ se pueden apreciar las vibraciones o modos normales de vibración correspondientes a metales. En esta región aparece la contribución del molibdeno, y como se mostrará más adelante en este capítulo, aparecerá también, hierro y cromo correspondientes al desgaste del acero del tazón o contenedor del molino y a los balines que se utilizan para la molienda.

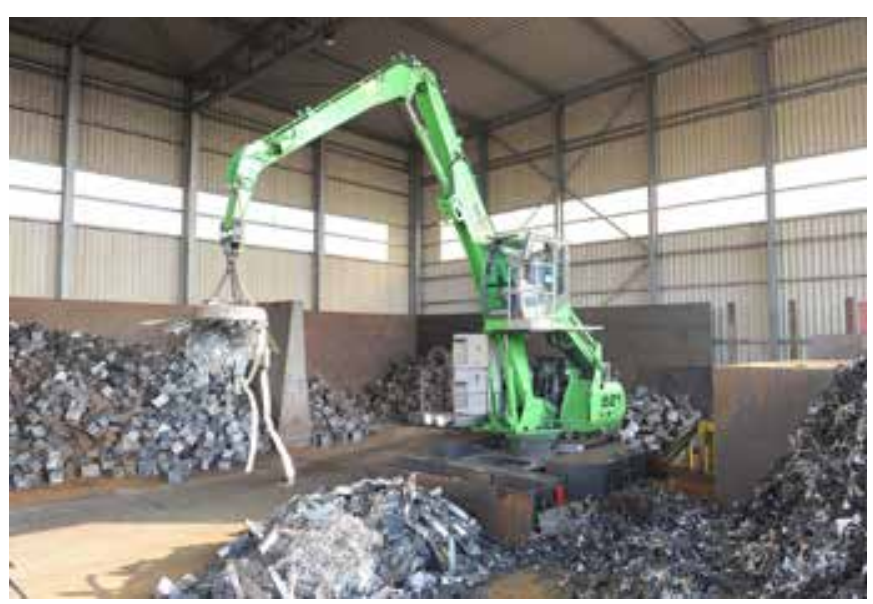



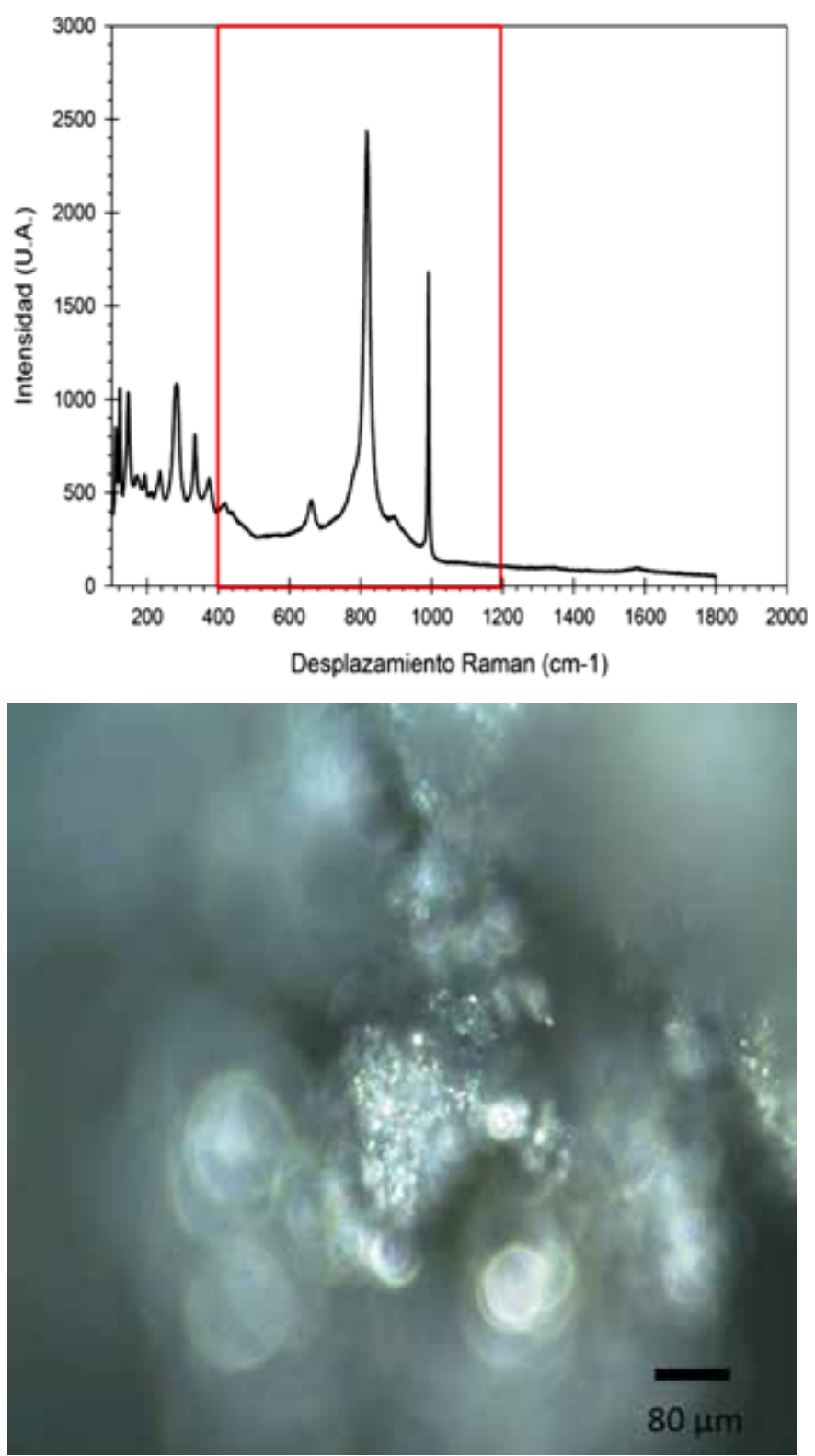

Figura 1. a) Espectrograma micro Raman típico de carburo de molibdeno $\mathrm{Mo}_{2} \mathrm{C}$ utilizando un espectrómetro micro Raman Xplora BX41.b) Microfotografía a 10X de una muestra de $\mathrm{Mo}_{2} \mathrm{C}$ obtenida sin tratamiento previo.

La figura 2 presenta los resultados obtenidos de la muestra de $\mathrm{Mo}_{2} \mathrm{C}$ tratada mediante aleado mecánico por un periodo de $8 \mathrm{hrs}$, el material de carburo presentan una reducción de tamaño apreciable respecto a la muestra a 0 hrs de molienda. En las imágenes obtenidas, utilizando un microscopio óptico, se observa un cambio muy pequeño en la estructura de los polvos conforme aumenta el tiempo de molienda (Figura 2), estos datos son confirmados mediante espectroscopía micro Raman, observándose las bandas 291.0, 346.0, 666.4, 819.5, 894.0 y $995.5 \mathrm{~cm}^{-1}$
(Figura 2), los mismos encontrados en la muestra en su estado inicial. La banda Raman a $819 \mathrm{~cm}^{-1}$ es la más intensa y corresponde a la vibración característica de carburo de molibdeno. Se observan además, aunque muy pequeña, una banda en $1580 \mathrm{~cm}^{-1}$ correspondiente a las vibraciones de la banda G (de grafito) [3].
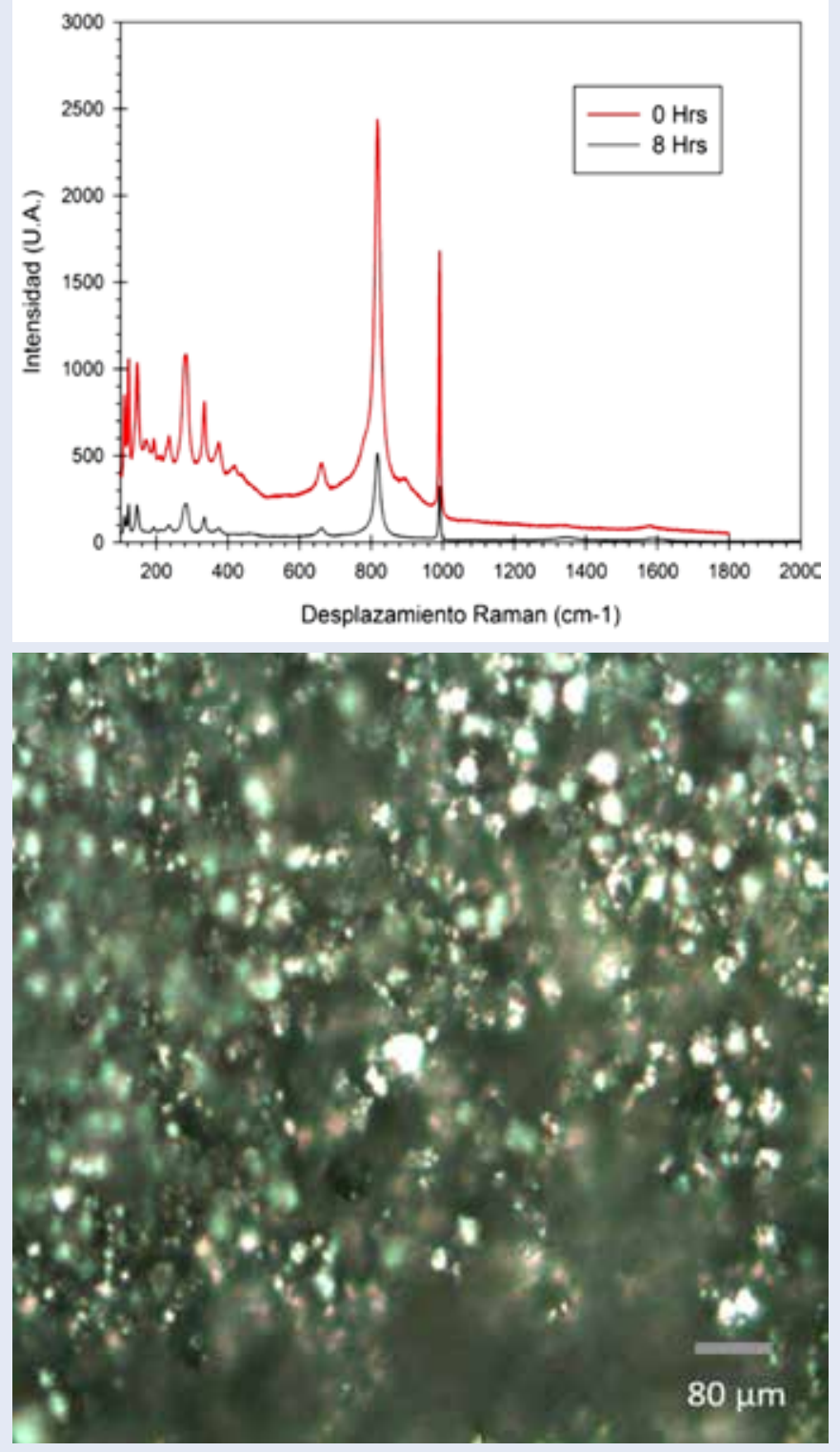

Figura 2. a) Espectrogramas micro Raman de carburo de molibdeno comparativo 0 hrs (línea continua roja) y

$8 \mathrm{hrs}$ de molienda. b) Microfotografía a 8 hrs de carburos de molibdeno observada con un objetivo 10X, muestra un tamaño promedio de $5.33 \mu \mathrm{m}$ de diámetro.

El espectro de esta muestra es característico al presentado por el $\mathrm{MoO}_{3}$ puro cristalino con las bandas Raman características en 118, 131, 159, 201, 220, 248, $287,340,370,382,474,668,821$ y $998 \mathrm{~cm}^{-1}$ [4] . Mientras que las muestras a $24 \mathrm{hrs}$ de molienda presentan una 
reducción de tamaño respecto a las muestras 0 hrs de molienda (Figura 3). Concomitando los espectros Raman presentan la aparición de las bandas a $1345.5 \mathrm{~cm}^{-1}$ y 1598.8 $\mathrm{cm}^{-1}$ asociadas al desorden de productos carbonosos (bandas D) y tangencial de los productos grafitizados (bandas G) respectivamente [5]. La banda D es indicadora de la presencia de defectos, de manera que de la relación de intensidades entre las bandas $D$ y $G$ puede extraerse información sobre el número de defectos [6].
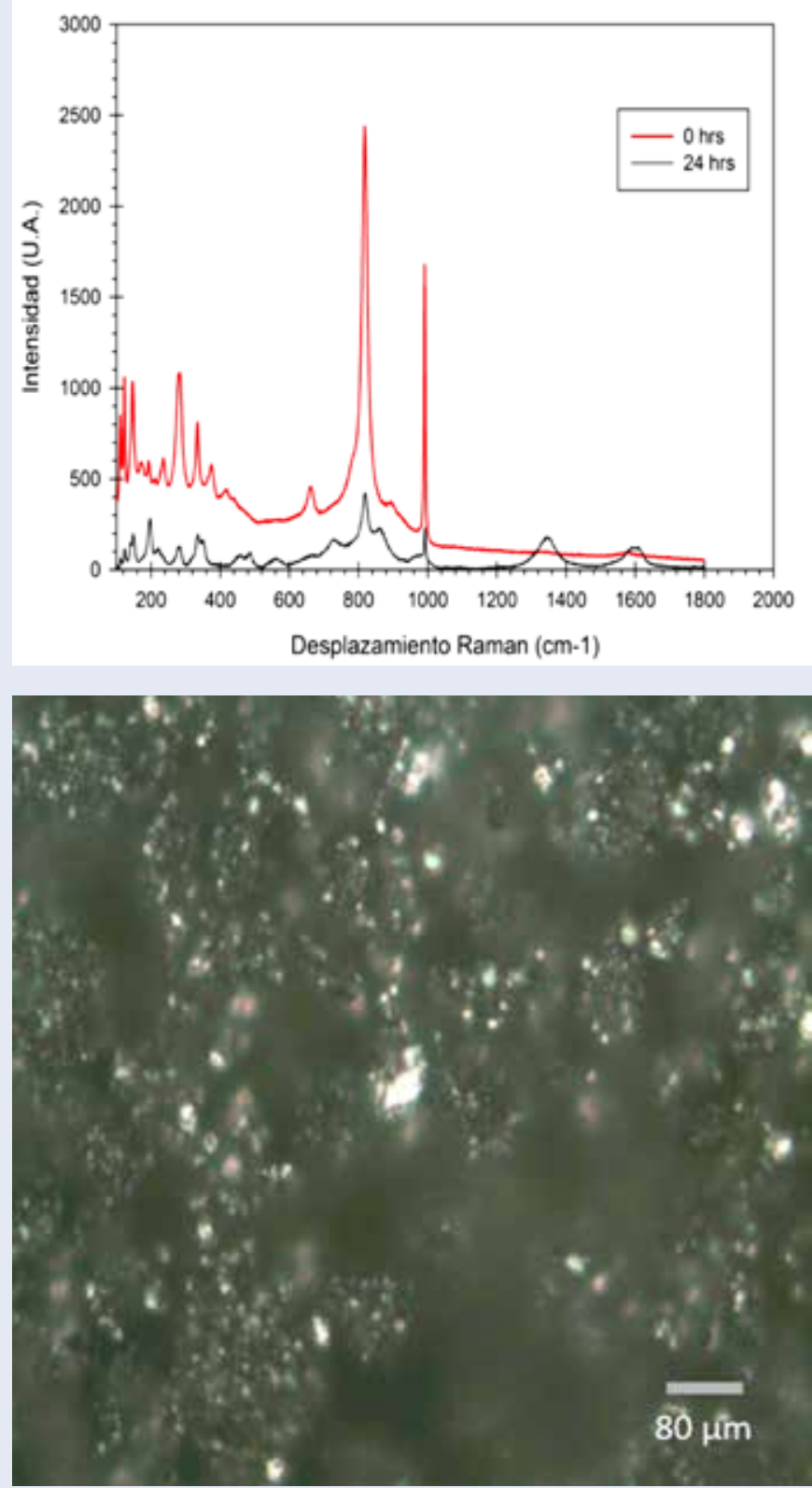

Figura 3. a) Espectrogramas micro Raman de carburo de molibdeno comparativo $0 \mathrm{hrs}$ (línea continua roja) y

24 hrs de molienda. b) Microfotografía a 10X de carburos de molibdeno observada con un objetivo 10X, donde se presentan tamaños de grano del orden de $2.34 \mu \mathrm{m}$.
En la figura 4 se pueden observar las bandas Raman de los carburos de molibdeno a $72 \mathrm{hrs}$ de molienda donde son más significativos los desplazamientos en las bandas a $850.2 \mathrm{~cm}^{-1}$ y $732.7 \mathrm{~cm}^{-1}$ así como los óxidos en la muestra entre $500 \mathrm{~cm}^{-1}$ y $700 \mathrm{~cm}^{-1}$ y además de las contribuciones de las bandas del carbono, mediante espectroscopía Micro Raman se puede observar que la intensidad de las bandas Raman $995.5 \mathrm{~cm}^{-1}, 894.0 \mathrm{~cm}^{-1}, 819.5 \mathrm{~cm}^{-1}, 666.4 \mathrm{~cm}^{-1}, 346.0$ $\mathrm{cm}^{-1}$, así como la aparición de la banda Raman a $778.7 \mathrm{~cm}^{-1}$ y el desplazamiento de las bandas a $894.0 \mathrm{~cm}^{-1}, 666.4 \mathrm{~cm}^{-1}$ y $778.7 \mathrm{~cm}^{-1}$ donde las bandas a $995.5 \mathrm{~cm}^{-1}$ y $778.7 \mathrm{~cm}^{-1}$ pueden ser asociadas a los estiramientos simétricos $\mathrm{y}$ anti simétricos de O-Mo-O, es decir, v ( O-Mo-O) [6, 7].
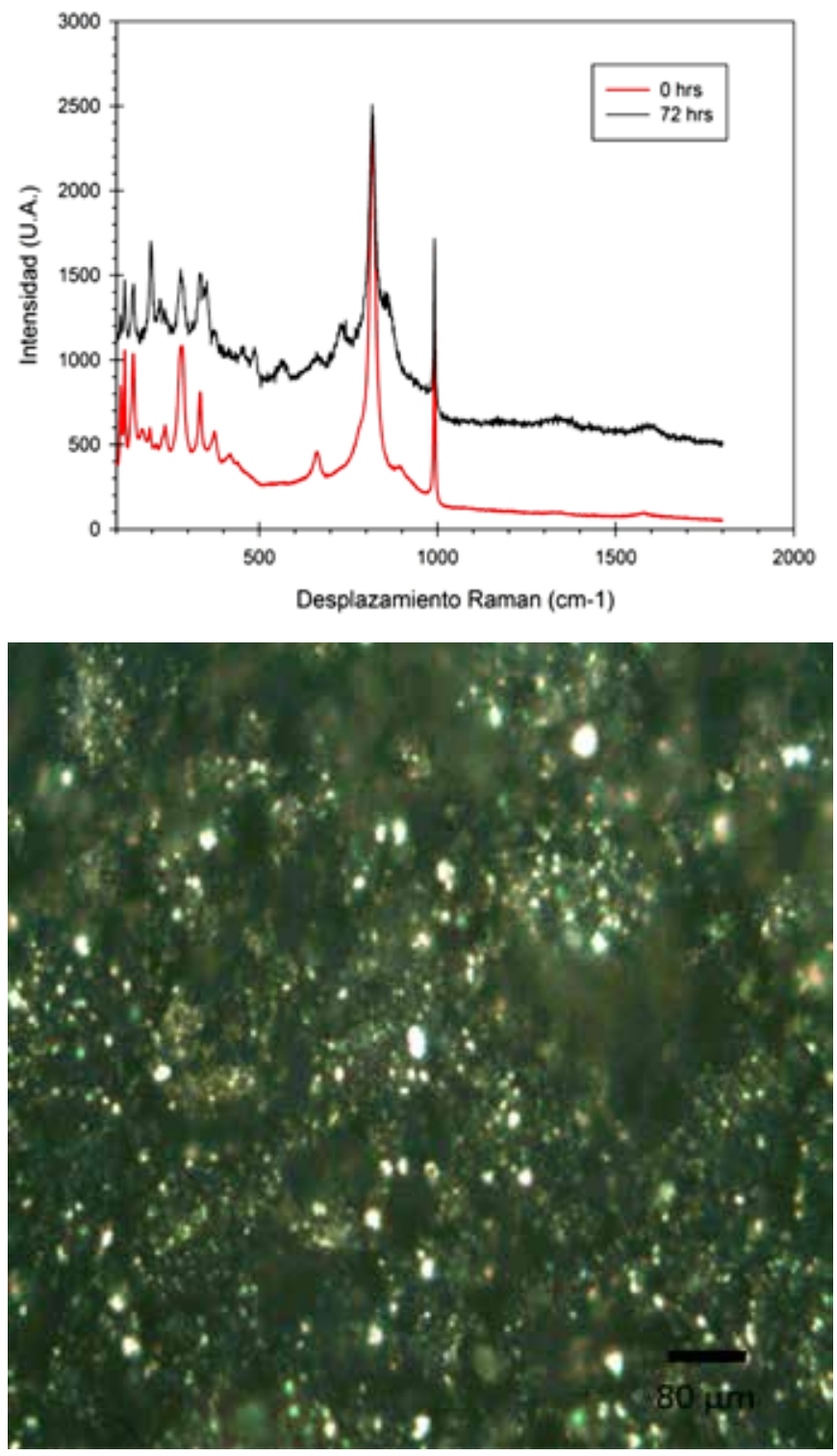

Figura 4. Espectrogramas micro Raman de carburo de molibdeno comparativo 0 hrs (línea continua roja) y 72 hrs de molienda. 


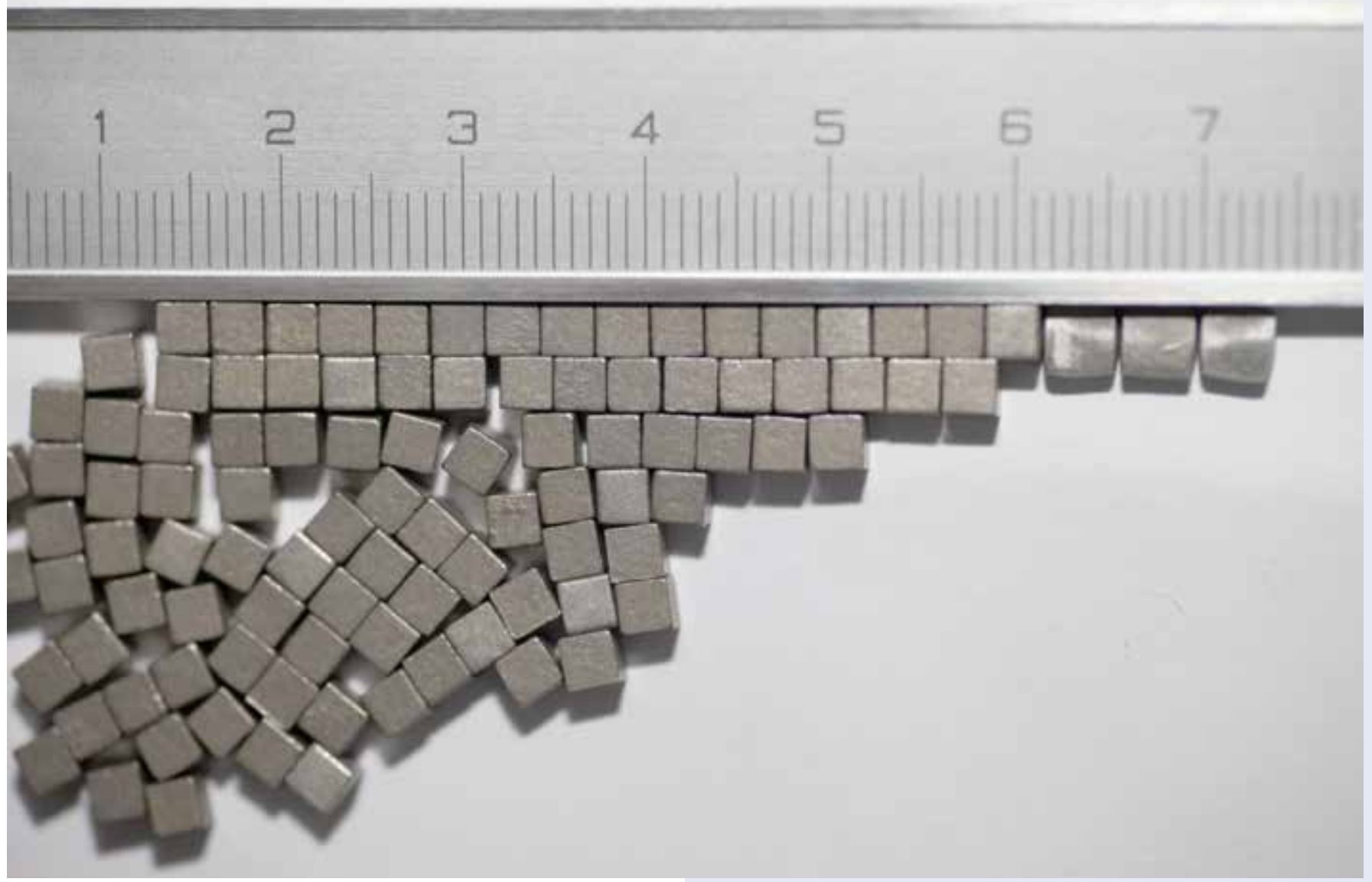

\section{Análisis de resultados}

Los resultados obtenidos mediante el microscopio óptico muestran una disminución en el tamaño de grano en función del tiempo de molienda, este comportamiento puede ser descrito en leyes de potencia, $y(t)=a t^{b}$ (Figura 5). En la figura 5 se observan dos regiones de tiempos, en donde el cambio en el tamaño de grano sigue una ley de potencia muy particular. El tiempo crítico, el cual marca la frontera entre los dos comportamientos es de aproximadamente $34 \mathrm{hrs}$ y 22 minutos. La primera región presenta una pendiente en una gráfica log-log, es decir, un exponente de -0.48 , mientras que para tiempos de molienda superiores a 34 hrs y 22 minutos, un comportamiento con potencia de -1.1 se hace presente. Esto es, la energía cinética que presentan los balines del sistema produce una molienda más eficiente, así también una disminución del tamaño de grano al incrementar el tiempo de molienda del material en el contenedor. Por otra parte, las bandas vibracionales de espectroscopía Raman presentan un desplazamiento hacia menores valores de desfasamiento Raman conforme el tiempo de molienda se incrementa, esto hace pensar que existe una buena correlación entre los resultados de espectroscopía Raman y los correspondientes a los tamaños de grano obtenidos por las mediciones de micrografías.

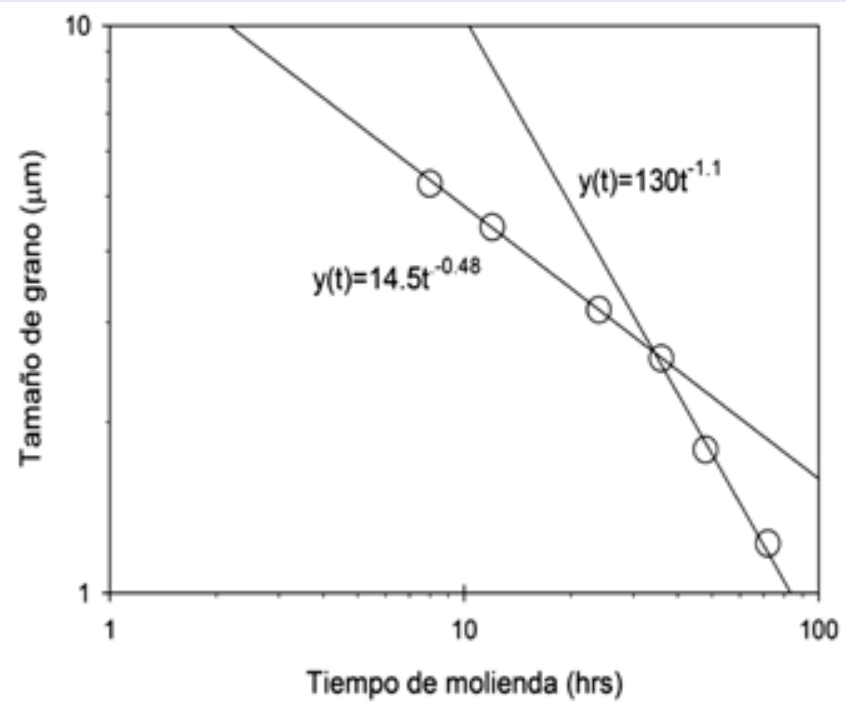

Figura 5. Gráfica con valores promedio de tamaño para cada muestra con respecto a los distintos tiempos de molienda mostrando la disminución de tamaño de grano. Las líneas continuas corresponden a las leyes de potencia $y(t)=a t^{b}$. 
La figura 6 muestra el desplazamiento de la banda característica de carburos de molibdeno $\mathrm{Mo}_{2} \mathrm{C}$ a medida que se incrementa el tiempo de molienda. La línea contínua que aparece en la figura 6 corresponde a un ajuste lineal $(y(t)=a t+b)$, siendo la pendiente de la recta igual a 0.035 , y el valor en el eje vertical a 819.5. Esto es, la banda del espectro Raman de $819.5 \mathrm{~cm}^{-1}$, característica del carburo de molibdeno, presenta un desplazamiento hacia valores de mayor energía, a medida que el tiempo de molienda aumenta, esto es al incrementarse la energía cinética de los balines, así como también se aprecian cambios muy marcados en el valor de la pendiente concomitando, observándose una disminución del tamaño de grano. Esto se ve reforzado por el desplazamiento de las bandas secundarias que se presentan a $666.8 \mathrm{~cm}^{-1}, 775.0 \mathrm{~cm}^{-1} \mathrm{y}$ $995.0 \mathrm{~cm}^{-1}$ conforme aumenta el tiempo de molienda, siguiendo un comportamiento lineal correspondiente a cambios en las bandas características del $\mathrm{Mo}_{2} \mathrm{C}$ así como apariciones de las bandas relacionadas al enlace O-Mo-O.

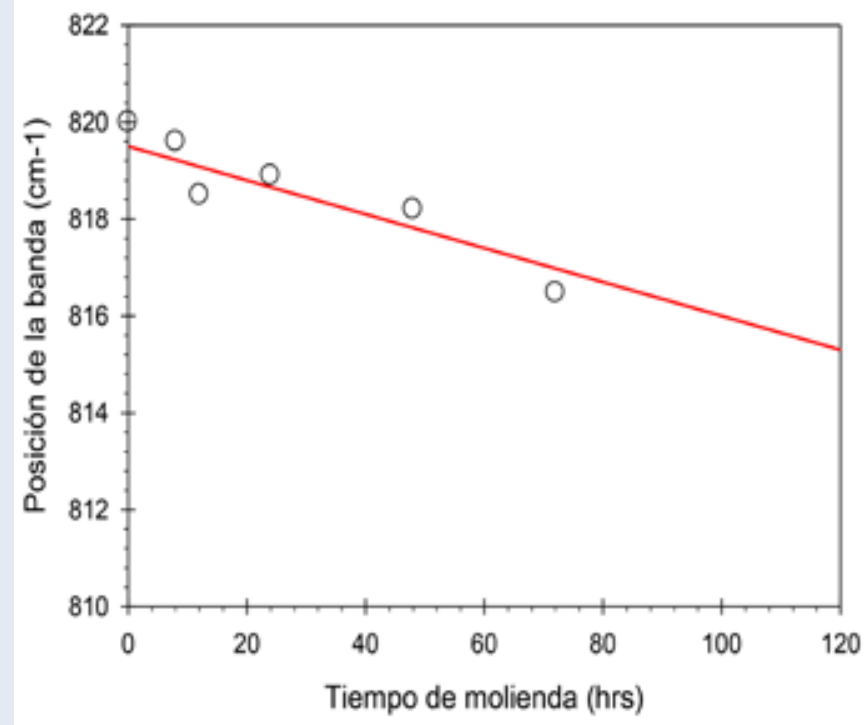

Figura 6. Desplazamiento de la banda de $819.5 \mathrm{~cm}^{-1}$ en función del tiempo de molienda para el compuesto carburo de molibdeno.

\section{CONCLUSIONES}

En este trabajo se llevó a cabo un estudio experimental del efecto de tamaño de carburos de molibdeno en función del tiempo de molienda, utilizando la técnica experimental de molienda mecánica en atmósfera de gas inerte, argón en nuestro caso. Las muestras obtenidas de carburo de molibdeno fueron caracterizadas mediante microscopía óptica y espectroscopía micro Raman. A medida que aumentaba el tiempo de molienda, se observaron disminuciones en los tamaños de frontera de grano, permitiendo llevar a cabo una correlación entre el tiempo de molienda y los cambios en la frontera de grano, mediante leyes de potencia. De esta manera se determinaron dos regiones principales de los tamaños promedios de las partículas, por medio de microscopía óptica. Siendo el tiempo critico en el cual se llevaba a cabo un cambio en el valor de la pendiente (en loglog, corresponde al exponente) de 34 hrs y 22 minutos. Mientras que por medio de espectroscopía Raman, se observaron cambios en los valores de las bandas principal y secundarias. Las tres bandas estudiadas presentan un comportamiento lineal en función del tiempo de molienda empleado en las mediciones experimentales. Los cambios presentes en las vibraciones Raman de las tres bandas principales fueron correlacionados a la disminución del tamaño de grano observado mediante microscopía óptica y a su vez con el cambio en el área superficial de las micropartículas de carburo de molibdeno.

\section{AGRADECIMIENTOS}

Luis Iván Serrano Corrales, Keren Hapuc Gutiérrez Acosta y Felipe Barffuson Domínguez agradecen al CONACYT por el apoyo económico.

\section{BIBLIOGRAFÍA}

[1] D. R. Askeland y P.P. Fulay, Ciencia e ingeniería de los materiales. Cengage Learnig, 2da Ed., 624 p., 2010.

[2] L. Díaz Barriga Arceo, E. Orozco, H. Mendoza-León, E. Palacios González, F. Leyte Guerrrero y V. Garibay Febles, «Nanostructures obtained from a mechanically alloyed and heat treated molybdenum carbide,» J. Alloys Compounds, Vol. 434-435, pp. 799-802, 2007.

[3] L. Ni, K. Kuroda, L.-P. Zhou, K. Ohta, K. Matsuishi y J. Nakamura, «Decomposition of metal carbides as an elementary step of carbon nanotube synthesis,» Carbon, Vol. 47, pp. 3054-3062, 2009.

[4] S.B. Xie, K.D. Chen, A.T. Bell y E. Iglesia, «Structural characterization of molybdenum oxide supported on zirconia,» J. Phys. Chem. B, Vol. 104, pp. 10059-10068, 2000.

[5] C. Domingo y G. Santoro, «Espectroscopía Raman de nanotubos de carbono,» Opt. Pura y Apl., Vol. 40, no 2, pp. 175-186, 2007.

[6] Tian-cun Xiao, Haitao Wang, Jianwen Da, Karl S. Coleman, and Malcolm L. H. Green, "Study of the preparation and catalytic performance of molybdenum carbide catalysts prepared with $\mathrm{C}_{2} \mathrm{H}_{2} / \mathrm{H}_{2}$ carburizing mixture". Journal of Catalysis 211, 183-191(2002).

[7] Zheng Liu, Lin Dong, Weijie Ji and Yi Chen, "Raman spectroscopic studies on tetragonal $\mathrm{ZrO} 2$ supported $\mathrm{MoO}_{3}$ and $\mathrm{ZnO}-\mathrm{MoO} 3$ systems". J. Chem. Soc., Faraday Trans., 94(8), 1137-1142 (1998).

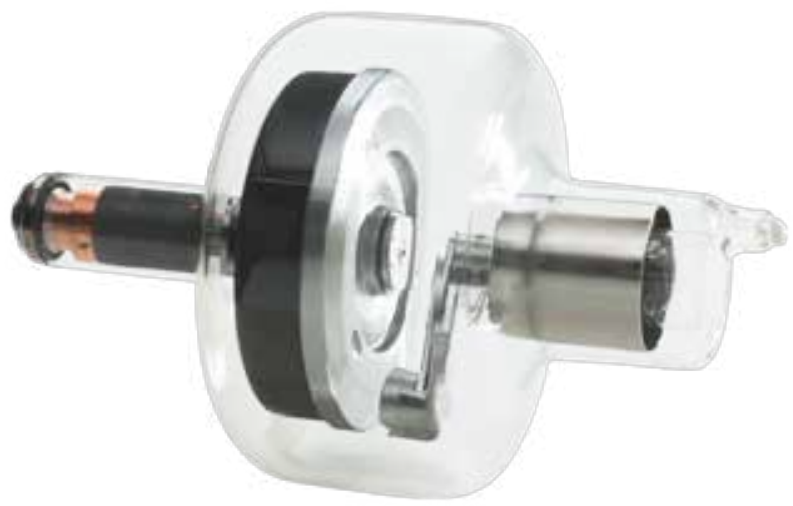

